\title{
Development of arabic sign language dictionary using 3D avatar technologies
}

\author{
Ahmed H. Aliwy, Ahmed A.Alethary \\ Department of Computer Science, Faculty of Computer Science and Mathematics, University of Kufa, Iraq
}

\begin{abstract}
Article Info
Article history:

Received Jun 2, 2020

Revised Aug 4, 2020

Accepted Aug 20, 2020

\section{Keywords:}

3D animation

Arabic sign language

Dictionaries

HamNoSys

Machine translation system

ABSTRACT

The arabic sign language (ArSL) is the natural language of the deaf community in Arabic countries. ArSL suffers from a lack of resources such as unified dictionaries and corpora. In this work, a dictionary of Arabic language to ArSL has been constructed as a part of a translation system. The Arabic words are converted into hamburg notation system (HamNoSys) using eSign editor Software. HamNoSys was used to create manual parameters (handshape, hand orientation, hand location, and hand movement), while non-manual parameters (facial expressions, shoulder raising, mouthing gesture, head tilting, and body movement) added by using (mouth, face, and limbs) in the eSign editor software. The sign then converted to the sign gesture markup language (SiGML) file, and later 3D avatar interprets the SiGML file scripts to the animated sign. The constructed dictionary has three thousand signs; therefore, it can be adopted for the translation system in which written text can be transformed into sign language and can be utilized for the education of deaf people. The dictionary will be available as a free resource for researchers. It is hard and timeconsuming work, but it is an essential step in machine translation of whole Arabic text to ArSL with 3D animations.
\end{abstract}

This is an open access article under the CC BY-SA license.

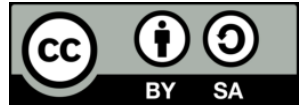

\section{Corresponding Author:}

Ahmed H. Aliwy

Department of Computer Science

Faculty of Computer Science and Mathematics

University of Kufa, Iraq

Email: ahmedh.almajidy@uokufa.edu.iq

\section{INTRODUCTION}

Sign language (SL) is the natural language of the deaf community. It consists of Manual Features (MF) and Non-Manual Features (NMF). The MF can be performed with both hands or with one hand in different shapes, locations, orientations, and movements, while NMF can be performed by facial expressions and movement of the body to embody the meaning of words and terms of a particular significance [1, 2]. In SL, the sign is specific hand shapes and movements that are associated with facial expressions to express the meaning. Also, SL is not universal, where every country has its sign language, which differs in syntax and grammar from one country to another [3, 4]. SL, which used in America, is identified as American Sign Language (ASL), but the used SL in India is identified as Indian Sign Language (ISL) [5] while in Arab countries identified as Arabic Sing Language (ArSL) [6]. Plus, ArSL is not universal; alternatively, it varies from one country to another and maybe differs in the same country. In Arab countries, there are Saudi, Iraqi, Moroccan, and Egyptian of the used sign languages [7]. In 1999, the first part of the unified dictionary of ArSL was published by The League of Arab States (LAS) and the Arab League Educational, Cultural and Scientific Organization (ALECSO) to unify the Arabic sign language (ArSL) and in 2007 the second part of 
the dictionary was published [8]. Deaf people in Arabic countries have a set of difficulties because of a few services are available to the deaf society. It has problems to access essential information or receive an education, communicate with the hearing community, and engage in community activities.

Several of work has been done of construction of sign language dictionary in worldwide. These dictionaries are prepared for SL in several countries like America, Spain, and Britain. The dictionaries have been constructed using human video or 3d avatar. Buttussia et al. [9] developed a structure of a dictionary using 3D animations for Multilanguage with excellent search functionality, and authoring mechanisms to expand the dictionary with new languages. thitsos et al. [10] created an American Sign Language (ASL) Video dataset, a thousand public videos of distinct ASL signs have been generated. The dataset can be used in computer vision and machine learning methods, especially for analyzing gestures and human communication. Gutierrez-Sigut et al. [11] developed a Spanish Sign Language lexical database. Its free online tool that consists of 2400 Videos. The database is available through a graphical interface to facilitate the searching process. Goyal [12] developed the synthetic animation dictionary for Indian Sign Language. 1818 sign has been generated. Sign Editor has been adopted for the generated HamNoSys and then converted to the SiGML file. Nagashima et al. [13] described a Japanese sign language dictionary for medical usage with 3D animation for showing the sign motions. The dictionary presented two types of searches: search by a description of sign language and search by Japanese keyword. For ArSL most of the dictionaries have been constructed in the form of books which is out-of-date now in the era of computerization. A few sign language dictionaries have been developed for automatic handling of ArSL sign language. Elhadj et al. [14] constructed a 3D animation dictionary for Saudi Sign Language using eSign projects. Hundreds of signs have been constructed and validated. The Islamic domain has been chosen for creating this dictionary. AlQallaf [15] developed a web-based bidirectional and bilingual video dictionary system. The system provided a visual interface to allow users to translate from an Arabic Sign language to an American Sign Language. Hamzah et al. [7] constructed a dictionary of images as a part of a translation system from the Arabic language to ArSL. The dictionary contains about 3200 signs. In the current work, an Arabic language to the ArSL dictionary is constructed as a part of a 3D animation system (3D animation dictionary for the unified ArSL). It the biggest dictionary for ArSL in this field.

\section{MATERIALS AND METHODS}

\subsection{HamNoSys}

HamNoSys is a phonetic transcription system was built in 1985 at the University of Hamburg in Germany. It designed based on the Stokoe Notation system and can be utilized for any sign language [16]. This notation was developed for a linguistic representation of signs such as sign generation, machine translation, and construction of SL dictionaries $[17,18]$. The Hamburg sign language notation system is also a basis for a set of avatar controls. HamNoSys involves more than 210 symbols. It is a language-independent notation system (any sign language can be represented); therefore, it can be accepted internationally. HamNoSys are describing a single sign using terms of parameters. These parameters are described in the order (symmetry parameters, non-manuals expression, handshapes, palm orientation, location, and movement) [19]. Figure 1 shows the structure of HamNoSys.

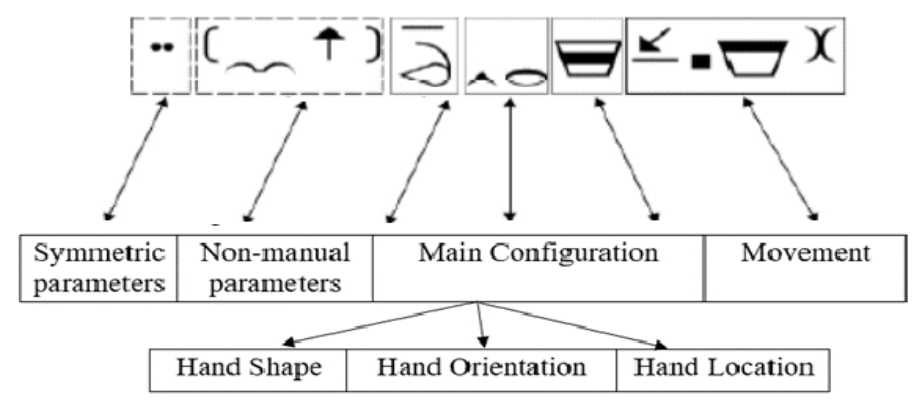

Figure 1. Structure of HamNoSys

\subsection{SiGML}

SiGML was initially produced in a ViSiCAST project [20] as a vital part of a model for natural language to the sign animation system. The main objective of SiGML is to support the interpretation of 
signing gestures in a way enabling them to be animated in live-time using an avatar. It is an XML scripting language, and it was built based on HamNoSys [21, 22]. It allows adding some physical features with a higher degree of precision than HamNoSys, but creating signs using SiGML is a complicated, hard and timeconsuming task. However, the semantic relationship between HamNoSys and SiGMLis lossless: HamNoSys can be translated into SiGML, and SiGML sign can be translated back into HamNoSys no information is wasted in this process [23].

\subsection{Esign software}

ESign software has two programs: eSign Editor and JASigning Player. ESign Editor was created by the eSIGN project [24] to let us generate signs in forms of HamNoSys and save it to the SiGML file. It possesses many advantages, such as functional movements and several handshapes, facial expressions, body movement, mouth gestures, and long sentences that can be signed; however, it requires experience in HamNoSys. Figure 2 shows the eSIGN Editor Interface. It allows us to add a new sign while the JASigning player software was designed to test the sign created by eSign editor [25].

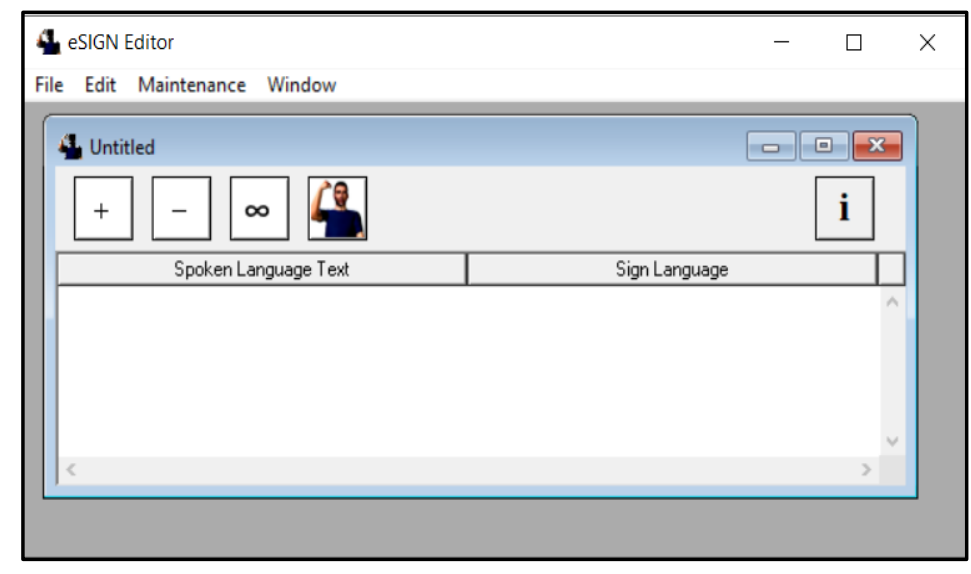

Figure 2. eSIGN Editor Interface

\section{CONSTRUCTION OF THE PREPOSING DICTIONARY}

Spoken language dictionaries such as the Oxford English Dictionary and Collins English Dictionary have generally used the listing words primarily by lemmas. For example: walk, walks, walking are listed in the Oxford Dictionary under lemmas walk because it is a canonical form while the other forms can be derived [26]. For example, مدارس [schools], مدرستكم [your school], and المدرسة [the school] all these forms considered to be variant forms of the lemma مدرسة [school]. However, the sign language dictionaries need not be arranged any differently. In the case of composite words that are the combination of two or more words and have to perform by one sign, the two lemmas will be taken. For example, كانون الثاني [January] is made up of one signs so arranged as كانون+ثاني [January].

A dictionary of Arabic word to ArSL constructed using eSign editor software, which uses HamNoSys as a notation system. The signs designed based on the unified Arab dictionary produced by The League of Arab States (LAS) and the Arab League Educational, Cultural, and Scientific Organization (ALECSO). Figure 3 shows the steps of the dictionary construction.

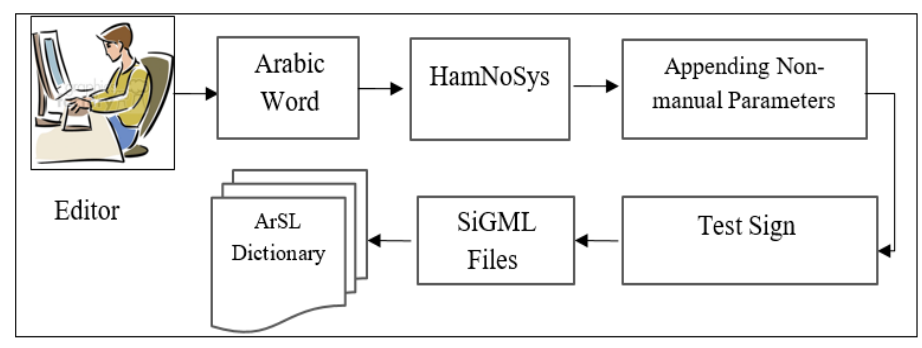

Figure 3. Steps of the dictionary construction 


\subsection{Adding a new word}

For each Arabic word, anew file will be created for this word. HamNoSys palette will appear, as shown in Figure 4.

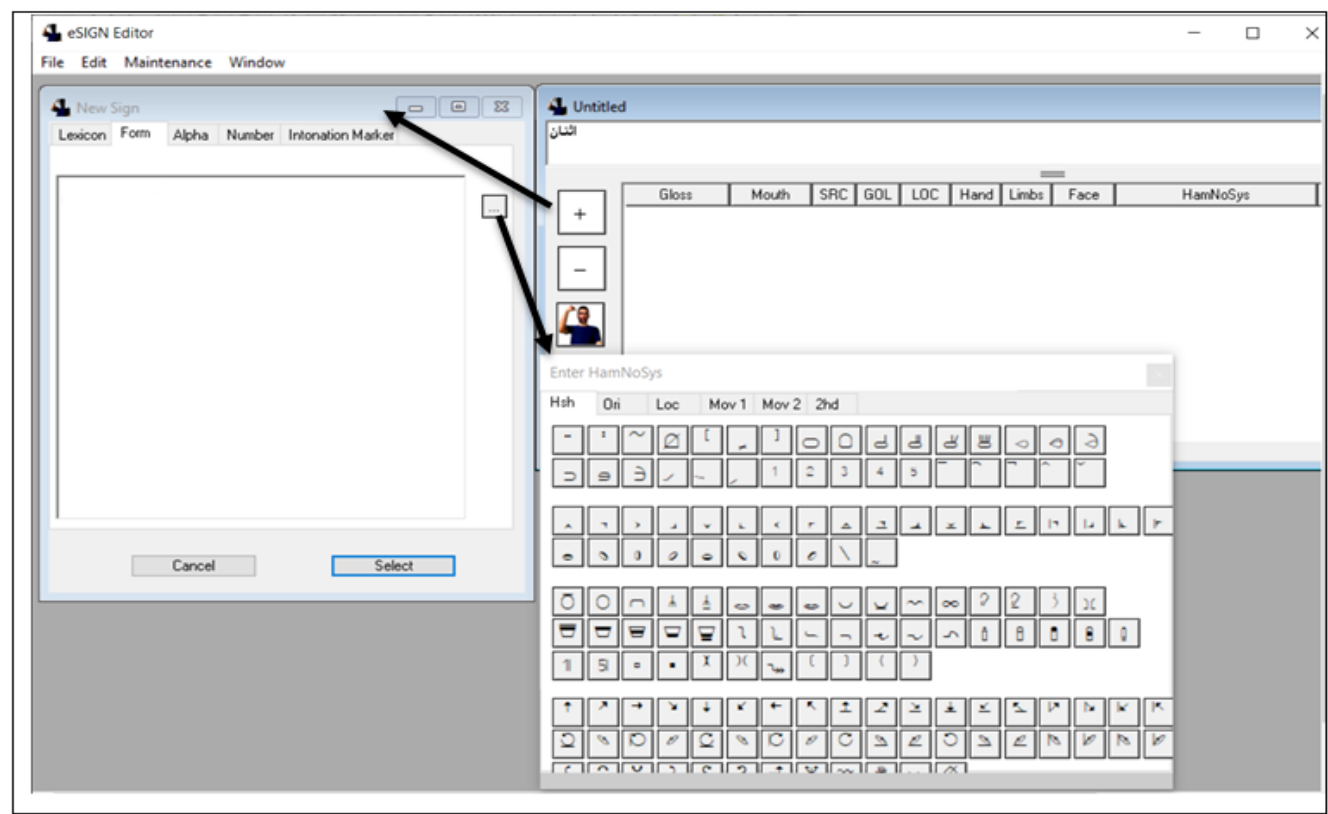

Figure 4. Appending new word

\subsection{Entering HamNoSys}

The structure of the HamNoSys has been illustrated. The HamNoSys of the sign can be created by selecting the handshape, the hand orientation, the hand location, and the hand movement from the HamNoSys input palette. Table 1 shows the steps for generating HamNoSys for the word اثنان [Two].

Table 1. Steps for generating HamNoSys for word إثنان [Two]

\begin{tabular}{ccc}
\hline Step & Hand Movement & Discerption \\
\hline 1 & & Handshape selected \\
2 & & Thumb finger added to the hand \\
3 & & Halm orientation to the back \\
4 & & Hand location at the Shoulder line \\
6 & & Hand contact with the body \\
7 & Straight hand movement to up
\end{tabular}

\subsection{Appending non-manual parameters}

After the description of manual parameters used HamNoSys, Appending the non-manual parameters (facial expressions, shoulder raising, mouthing gesture, head tilting, and body movement) using mouth, face, and limbs in eSign editor software. This step will add more realism to the sign. Figure 5 shows how to add mouth picture and eye gaze of the sign اثنان [Two]. Mouth picture indicates the description that refers to how the word pronounced. The اثنان [Two] is Pronounced as (ithnan). 


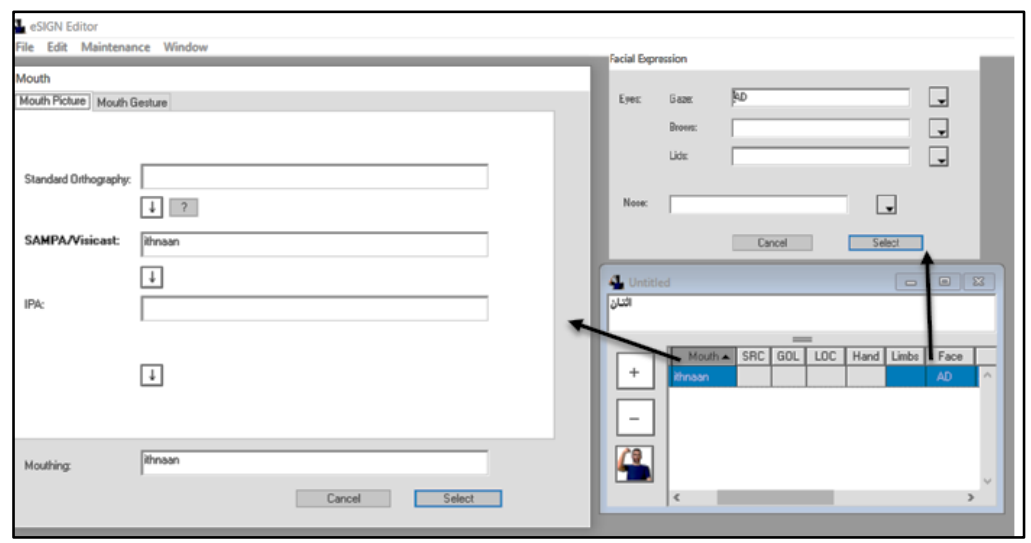

Figure 5. Adding mouth movement and eye gaze of the word Two

\subsection{Testing the sign}

After the description of manual and non-manual parameters, the sign will be tested to check its fitting using JASigning player software that was designed to test the sign created by eSign editor. as shown in Figure 6 .

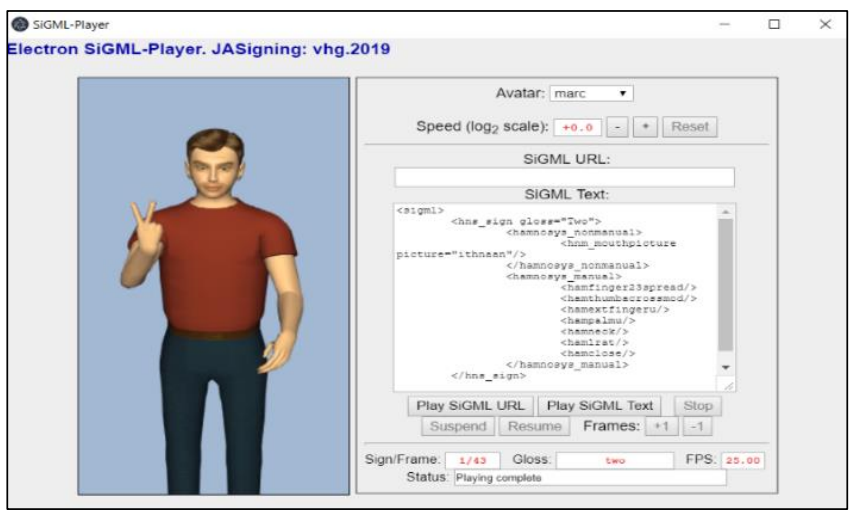

Figure 6. Test the sign two used JASigning player

\subsection{SiGML file generation}

Once the sign generated and tested correctly, it will be exported as a SiGML file script, and later 3D avatar interprets the file in online mode. Figure 7 shows a sample of SiGML file script for one word. For each generated SiGML file assigns an index number representing the name of this file. This index will be added to the dictionary beside the Arabic word, which is in the form of lemma.

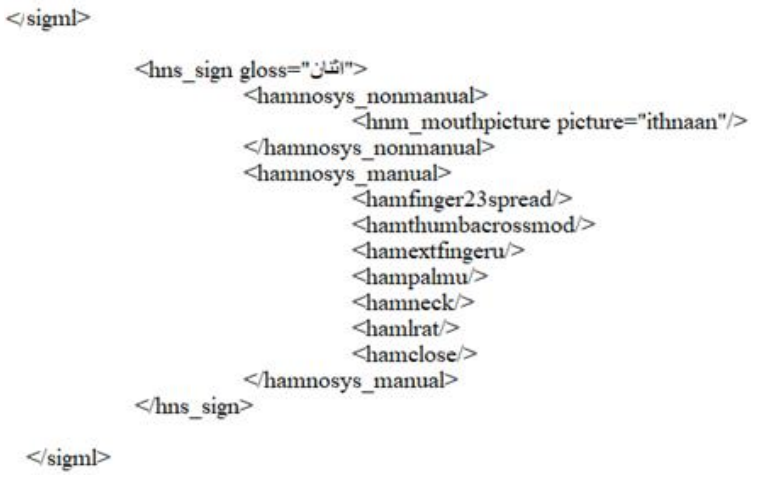

Figure 7. The SiGML file script for word اثنان [Two] 


\section{RESULTS AND DISCUSSION}

The dictionary is saved as a CSV file where the Arabic word is stored according to its lemma, and every word assigns an index number representing the name of the SiGML file. The dictionary contains about 3000 signs, which means it is the largest known ArSL dictionary. Its construction has consuming time and effort. The individual sign is coded during a broad range of SL phonological parameters, including manual parameters and non-manual parameters. Our dictionary was tested with multiple sentences of ArSL. These sentences are also experimented with a dictionary of videos. The result shows that animation processing is faster than video processing result from the loading of large files of videos for each sentence to a cache memory. Two experts of sign language have been asked to evaluate the quality of generated signs on the range from 1 to 5 for 100 sign selected randomly. The average rated was 4.3. The results are actually really encouraging and are meriting to be utilized for communication with deaf people. The dictionary can be adopted in the translation system of text to sign language and for the education of deaf people. Figure 8 shows a sample of images of the unified ArSL dictionary that used for signs generation where Table 2 shows an example of generated signs. Table 3 shows the comparing of the results of this research with others for ArSL [10,3].

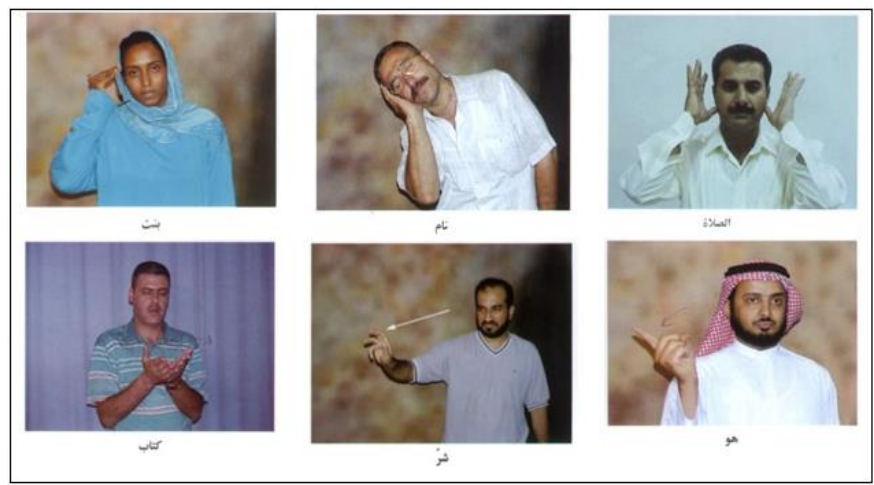

Figure 8. Sample of images from unified ArSL dictionary

Table 2. Samples of Generated Signs

\begin{tabular}{|c|c|c|c|c|}
\hline No & Word & Generated HamNoSys & Non-manual parameters & 3D avatar \\
\hline 1 & [girl] & 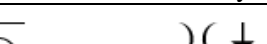 & - Mouth-picture (bent) & \\
\hline 2 & [صلاة [ Prayer] & $\uparrow$ & $\begin{array}{ll}\text { - } & \text { Mouth-picture (naam) } \\
\text { - } & \text { Eyes lids (closed) } \\
\text { - } & \text { Body (tilted right) } \\
\text { - } & \text { Head (tilted right) }\end{array}$ & \\
\hline 3 & نام[sleep] & & - Mouth-picture (salat) & \\
\hline
\end{tabular}




\begin{tabular}{|c|c|c|c|c|}
\hline No & Word & Generated HamNoSys & Non-manual parameters & 3D avatar \\
\hline 4 & كتاب [book] & $\stackrel{\leftarrow}{\leftarrow} \rightarrow 0^{\prime}$ & $\begin{array}{ll}- & \text { Mouth-picture (kitab) } \\
\text { - } & \text { Head (tilted forward) }\end{array}$ & \\
\hline 5 & شر[evil] & $\bar{\ominus} \wedge \circ \bigcirc \cdot)([\lrcorner щ, \curvearrowright E$ & $\begin{array}{ll}\text { - } & \text { Mouth-picture (shar) } \\
\text { - } & \text { Eyes brows (furrowed) } \\
\text { - } & \text { Eyes lids (narrowed) } \\
\text { - } & \text { Body (tilted left) }\end{array}$ & \\
\hline 6 & هو [he] & $\Leftrightarrow 70)\left[\begin{array}{l}\lambda \\
0\end{array}\right.$ & $\begin{array}{l}\text { Mouth-picture (huwa) } \\
\text { Eyes lids (left) }\end{array}$ & \\
\hline
\end{tabular}

Table 3. The comparing of the results of this research with others for ArSL

\begin{tabular}{cccc}
\hline The topic & Current research & Research [10] & Research [3] \\
\hline Technique & 3D avatar & 3D avatar & Images \\
Domain & All domain & Islamic domain & All domain \\
Number of generated sign & 3000 signs & A hundred of sign & 3200 signs \\
Country & Unified dictionary & Saudi Sign Language & Unified dictionary \\
\hline
\end{tabular}

\section{CONCLUSION}

In the current work, a new dictionary for ArSL has been developed using avatar technologies where each sign generated based on the unified Arab dictionary. Using the 3D avatar technologies is much better than using human video because of the least memory consumption, less conversion time, supported by a translation system and avatar can be varied according to choice. The dictionary can be adopted for the translation system in which written text can be transformed into sign language and can be utilized for the education of deaf people. For the construction of complete ArSL avatar technologies, a team of experts of SL, workers and programmers should be work together.

\section{REFERENCES}

[1] S. Goldin-Meadow and D. Brentari, "Gesture, sign, and language: The coming of age of sign language and gesture studies," Behavioral and Brain Sciences, vol. 40, pp. 1-17, Oct. 2017.

[2] H. Hafit, C. W. Xiang, M. M. Yusof, N. Wahid, and S. Kassim, "Malaysian sign language mobile learning application: A recommendation app to communicate with hearing-impaired communities," International Journal of Electrical and Computer Engineering, vol. 9, no. 6, pp. 5512-5518, 2019.

[3] W. N. Khotimah, T. Anggita, and N. Suciati, "Indonesian sign language recognition using kinect and dynamic time warping," Indonesian Journal of Electrical Engineering and Computer Science, vol. 15, no. 1, pp. 495-503, 2019.

[4] T. M. Angona et al., "Automated bangla sign language translation system for alphabets by means of MobileNet," Telkomnika (Telecommunication Computing Electronics and Control), vol. 18, no. 3, pp. 1292-1301, 2020.

[5] M. Kumar, "Conversion of Sign Language into Text," International Journal of Applied Engineering Research, vol. 13, no. 9, pp. 7154-7161, 2018.

[6] M. Brour and A. Benabbou, "ATLASLang MTS 1: Arabic Text Language into Arabic Sign Language Machine Translation System,” Procedia Computer Science, vol. 148, no. Icds 2018, pp. 236-245, 2019.

[7] H. Luqman and S. A. Mahmoud, "Automatic translation of Arabic text-to-Arabic sign language," Universal Access in the Information Society, vol. 0, no. 0, pp. 1-13, 2018.

[8] "Arabic sign language dictionary. http://www.menas y.com/."

[9] F. Buttussi, L. Chittaro, and M. Coppo, "Using Web3D technologies for visualization and search of signs in an international sign language dictionary," in Proceedings of the twelfth international conference on $3 D$ web 
technology - Web3D '07, 2007, vol. 2007, p. 61.

[10] V. Athitsos et al., "The American Sign Language Lexicon Video Dataset," 2008 IEEE Computer Society Conference on Computer Vision and Pattern Recognition Workshops, CVPR Workshops, 2008.

[11] E. Gutierrez-Sigut, B. Costello, C. Baus, and M. Carreiras, "LSE-Sign: A lexical database for Spanish Sign Language," Behavior Research Methods, vol. 48, no. 1, pp. 123-137, Mar. 2016.

[12] L. Goyal and V. Goyal, "Development of Indian Sign Language Dictionary using Synthetic Animations," Indian Journal of Science and Technology, vol. 9, no. 32, Aug. 2016.

[13] Y. Nagashima and K. Watanabe, "Medical Sign Language Dictionary with 3D Animation Viewer," The Second International Conference on Advances in Computation, Communications and Services, no. c, pp. 19-20, 2017.

[14] Y. O. M. Elhadj, Z. Zemirli, and B. Al-faraj, "Towards a unified 3D animated dictionary for Saudi sign language," in Proceedings of the International Conference on Advances in Computing, Communications and Informatics ICACCI '12, 2012, p. 910.

[15] A. H. Alqallaf, "Development of a Web-based Unified Arabic/American Sign Language Bilingual Dictionary," Journal of Engineering Research, vol. 6, 2018

[16] R. Kennaway, "Synthetic Animation of Deaf Signing Gestures," In International Gesture Workshop (pp. 146-157). Springer, Berlin, Heidelberg.2001,

[17] S. Diwakar and A. Basu, "A Multilingual Multimedia Indian Sign Language Dictionary Tool," Proceedings of the 6th Workshop on Asian Language Resources, pp. 57-64, 2008.

[18] K. Kaur and P. Kumar, "HamNoSys to SiGML Conversion System for Sign Language Automation," Procedia Computer Science, vol. 89, pp. 794-803, 2016.

[19] D. S. Sharma et al., "Automatic Translation of English Text to Indian Sign Language Synthetic Animations," NLP Association of India, no. December, pp. 144-153, 2016.

[20] J. A. Bangham et al., "Virtual signing: Capture, animation, storage and transmission - An overview of the ViSiCAST project," IEE Colloquium (Digest), no. 25, pp. 23-29, 2000.

[21] R. Elliott, J. Glauert, V. Jennings, and R. Kennaway, "An Overview of the SiGML Notation and SiGMLSigning Software System," In the Fourth International Conference on Language Resources and Evaluation, LREC 2004 (pp. 98-104), pp. 98-104, 2004.

[22] K. Ayadi, Y. O. M. Elhadj, and A. Ferchichi, "Prototype for Learning and Teaching Arabic Sign Language Using 3D Animations," 2018 International Conference on Intelligent Autonomous Systems, ICoIAS 2018, pp. 51-57, 2018.

[23] Z. Kang, "Spoken language to sign language translation system based on HamNoSys," ACM International Conference Proceeding Series, pp. 159-164, 2019.

[24] I. Zwitserlood, M. Verlinden, J. Ros, S. Van Der Schoot, and T. Netherlands, "SYNTHETIC SIGNING FOR THE DEAF: eSIGN," Proceedings of the conference and workshop on assistive technologies for vision and hearing impairment, CVHI. Vol. 2004, 2004.

[25] Q. L. Da, N. H. D. Khang, and N. C. Ngon, Converting the vietnamese television news into 3D sign language animations for the deaf, vol. 257. Springer International Publishing, 2019.

[26] J. Fenlon, K. Cormier, and A. Schembri, "Building BSL SignBank: The Lemma Dilemma Revisited," International Journal of Lexicography, vol. 28, no. 2, pp. 169-206, Jun. 2015.

\section{BIOGRAPHIES OF AUTHORS}

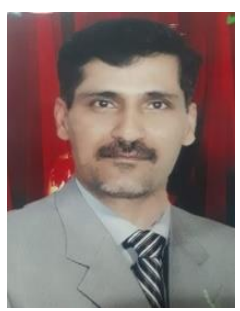

Mr. Ahmed Hussein Aliwy earned his PhD in the field of computer science from university of warsaw, Poland, 2013, He completed his M.sc in university of technology, Baghdad, Iraq, 2004 and BSc in Electrical Engineering, university of technology, Baghdad, Iraq, 1997. His research interests include NLP, text mining and what is located within this field of applications.

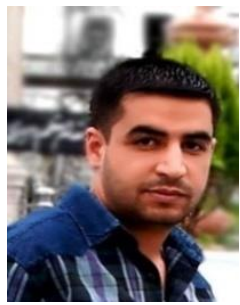

Mr. Ahmed Arif Jaber earned his BSc in the field of computer science from university of qadisiyah, diwaniya, Iraq, 2010, He completed his M.sc in university of kufa, najaf, Iraq, 2020. His research interests include Arabic NLP and what is located within this field of applications. 